\title{
Problems of creating autonomous navigation systems on geophysical fields
}

\author{
Vyacheslav Fateev ${ }^{1}$, Dmitrii Bobrov ${ }^{1}$, Murat Murzabekov ${ }^{1 *}$, and Ruslan Davlatov ${ }^{1}$ \\ ${ }^{1}$ All-Russia Research Institute of Physical and Radio Engineering Measurements, 141570, Moscow \\ region, Mendeleevo, Russian Federation
}

\begin{abstract}
Global navigation satellite systems, which provide high accuracy of navigation, in certain conditions (in tunnels, in closed rooms, in conditions of interference, etc.) have restrictions on their use. In this regard, in order to ensure "seamless" navigation in any conditions of the situation, it becomes necessary to develop new methods and means to increase the stability of navigation definitions. The article is devoted to the consideration of the problems of creating an integrated navigation system using measurements of the parameters of the Earth's gravitational and magnetic fields. Requirements for meters of parameters of geophysical fields and navigation charts are considered, a number of new navigation meters, new methods and means of preparing navigation charts are proposed. The ways of development of relativistic geodesy and the possibility of using the achievements of gravitational-wave astronomy in gravimetry are considered.
\end{abstract}

\section{Introduction}

The widely used global navigation satellite systems (GNSS) GPS, GLONASS, GALILEO, BeiDou provide high potential navigation definition accuracy, global coverage and navigation continuity. However, there are a number of situations in which the level of received GNSS signals is either below the threshold level, for example, in tunnels, canyons, enclosed spaces, or reception of signals is generally impossible (underground, under water, on other planets). In addition, the problem of working in conditions of natural and deliberate interference, including changes in the power of signals from spacecraft, up to their complete shutdown, does not lose its relevance. Modern requirements for the reliability and continuity of "seamless" navigation in conditions of interference and inaccessibility of GNSS signals require the development of new methods and means to increase the stability and reliability of navigation systems.

One of the way to increase the noise immunity of navigation solutions is to create an integrated navigation system based on the use of a correlation-extreme navigation system (CENS) operating on the anomalous Earth's gravitational field (EGF) and Earth's magnetic field (EMF). It should include an airborne strapdown navigation system (SINS) based on the use of accelerometers and gyroscopes, as well as sensors of the current parameters of

\footnotetext{
*Corresponding author: murzabekov@ vniiftri.ru
} 
the EGF and EMF. Since the spatial distributions of the characteristics of anomalous EGF and EMF near the Earth's surface are unique and rather stable, the measurements of the current values of the parameters of the EGF and EMF can be used for global navigation.

The most important property of the EGF is its stability over time. Methods of setting organized interference to SINS, as well as to onboard navigation sensors EGF and EMF are unknown.

\section{Assessment of the achievable accuracy of an integrated navigation system using measurements of the parameters of the EGF and EMF}

To assess the achievable accuracy of an integrated navigation system using a EGF, its mathematical modeling was carried out using a special model. In this case, joint measurements of SINS (with drift $1.8 \mathrm{~km} / \mathrm{h}$ ), gravity anomalies (GA) and gravitational gradient (GG) (SINS + gravimeter + gradiometer) were used. For modeling, GA maps with an average gradient of $4 \mathrm{mGal} / \mathrm{km}$ and GG with an average gradient of 30 Eotvos $/ \mathrm{km}$ were used, which corresponds to the foothill (hilly) area. Fig. 1a shows the simulation results based on GA measurements, Fig. 1b - according to measurements of the GG, Fig. 1c - when using GA and GG together.

The assessment of the prospective accuracy of navigation by the EMF was carried out for a typical area of the Atlantic Ocean with dimensions of $250 \times 500 \mathrm{~km}$ with anomalies from $-800 \mathrm{nT}$ to $+1200 \mathrm{nT}$ at the sea level (SINS + magnetometer). In this case, the daily variations in the EMF were set in the range of $6 \div 30 \mathrm{nT}$. The influence of natural noise in the form of magnetic storms was set in the range of $120 \div 150 \mathrm{nT}$. Simulation conditions: error of the EMF map - 10 $\div 100 \mathrm{nT}$, SINS drift - $1 \mathrm{mph}(1.8 \mathrm{~km} / \mathrm{h})$. The simulation results are shown in Fig. 1d.

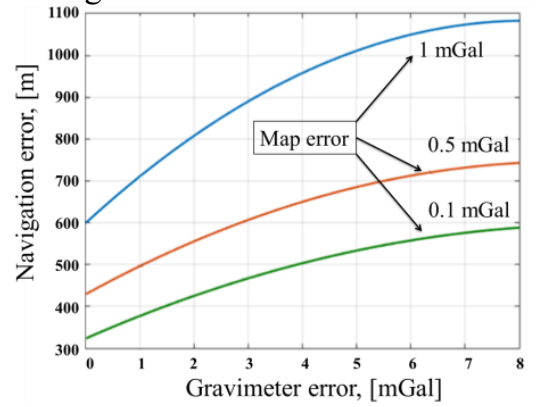

a)

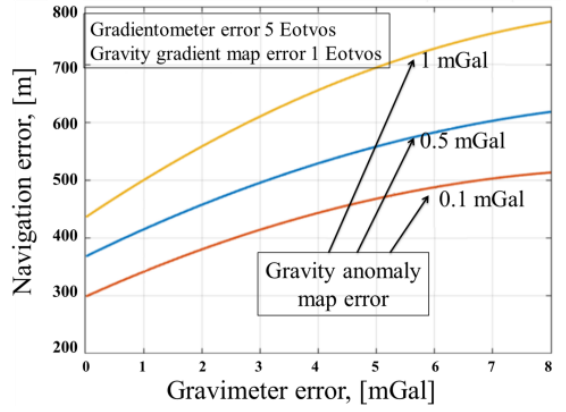

c)

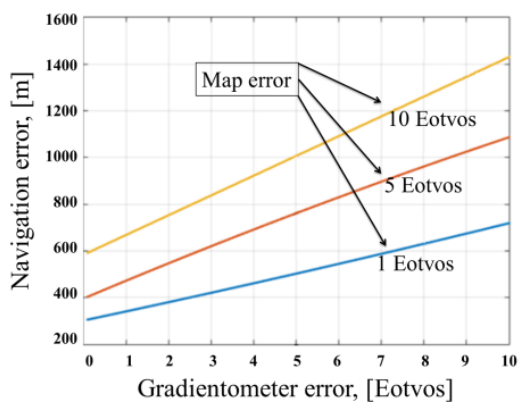

b)

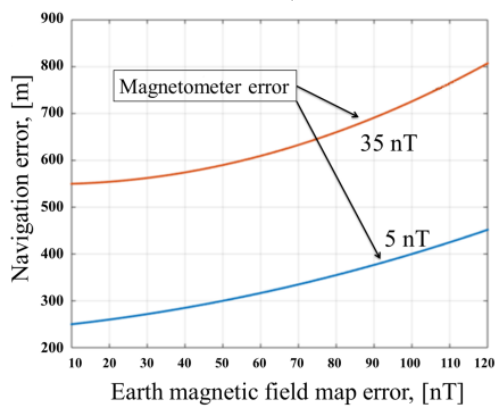

d)

Fig. 1. The results of modeling the error of the complex geophysical navigation system: a) by measurements of SINS + GA; b) by measurements of SINS + GG; c) by measurements of SINS + GA $+\mathrm{GG} ;$ d) by measurements of SINS + EMF 
Based on the modeling of the EGF navigation system, the following main results were obtained:

a) with an error of the on-board gravimeter of $1 \mathrm{mGal}$ and an error of the navigationgravimetric map of $0.05,0.1$ and $1 \mathrm{mGal}$, the navigation error is 380,505 and $710 \mathrm{~m}$, respectively;

b) with an error of the gravity gradient meter 5 Eotvos, as well as with a map error of 1 , 5 and 10 Eotvos, the navigation error was 510,760 and $1020 \mathrm{~m}$, respectively;

c) with the combined use of GA and GG, the navigation error is reduced by about $15 \%$ relative to the use of these meters separately.

Based on the simulation results, it is possible to formulate the requirements for the meters of the parameters of the EGF to ensure the accuracy of navigation of hundreds of meters on hilly terrain:

- for on-board gravimeters, the measurement error should be $0.5 \mathrm{mGal}$ or less;

- for gradiometers - no more than $1 \div 2$ Eotvos.

For the implementation of "seamless" navigation, which should provide an error commensurate with the accuracy of GNSS, the requirements are more stringent:

- for on-board gravimeters, the required error is $0.01 \mathrm{mGal}$ or less;

- for gradiometers, an error of 0.01 Eotvos or less is required.

When using the EMF, the currently achievable navigation errors are generally commensurate with the EGF and amount to hundreds of meters.

\section{The ground onboard layout for navigation in geophysical fields and its testing}

FSUE "VNIIFTRI" has created and tested an onboard model of a ground vehicle navigation system for geophysical fields - EGF and EMF. It includes an SINS, a magnetic field meter (magnetometer and magnetic gradiometer), and a small-sized onboard gravimeter developed at FSUE "VNIIFTRI".

At the first stage, navigational maps of the magnetic and gravitational fields parameters were created. For this, the results of measurements of the EGF and EMF parameters were processed on the selected section of the route of movement. At the stage of navigation of the mobile carrier, the correction of the onboard SINS drift was performed based on the measurement of geophysical parameters in real time.

When preparing navigation maps, measurements were carried out in the Moscow region. To create a map of free fall acceleration, measurements were done with a discreteness of 3-5 km along the route. Further, on the basis of a digital model of terrain heights and data on geological structures, high-precision interpolation was performed to increase the resolution of the map to $30 \mathrm{~m}$.

Fig. 2 shows the results of testing the layout. The actual and calculated trajectories of the vehicle are shown in Fig. 2a. Blue line - this is the real trajectory of the prototype, calculated from the measurements of the GNSS navigation receiver. Red line - trajectory obtained from SINS measurements. For 50 minutes of movement, SINS has accumulated a position determination error of more than $3 \mathrm{~km}$. Green Line - this is the result of the correction of the SINS trajectory based on the use of data of EGF and EMF parameters.

Fig. $2 b$ shows a graph of corrections of SINS readings in time. Correction intervals did not exceed 4 minutes. In general, when using information about the current EGF and EMF parameters, the navigation error on the route did not exceed $900 \mathrm{~m}$. 


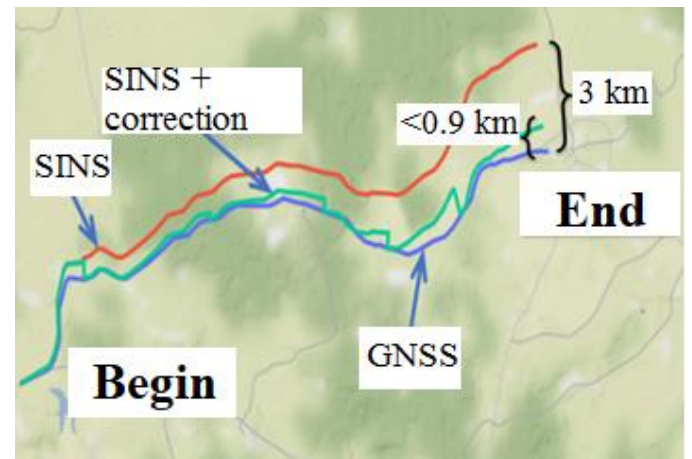

a)

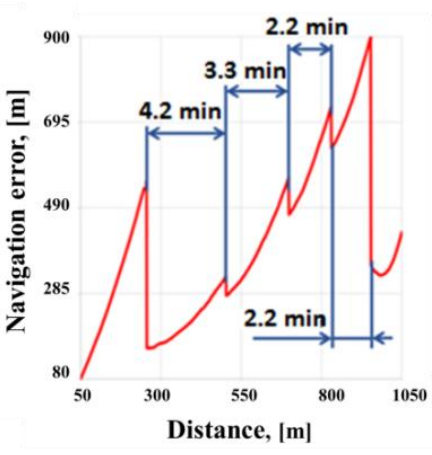

b)

Fig. 2. Test results of the ground onboard layout for navigation in geophysical fields: a) test route; b) example of correction the SINS

\section{Problems of creating gravimetric and magnetometric navigation systems}

\section{The main problems of gravimetric navigation systems:}

1) the problem of preparing high-precision maps of the parameters of the EGF. To solve it you need:

- increasing the degree and order of the EGF models to increase their spatial resolution;

- expanding the park and improving the accuracy of land, sea and air meters of the EFG for the preparation of regional and local navigation maps;

- increasing the accuracy of the elevation maps of large areas by $10 \div 100$ times, which requires the creation of a fundamentally new measuring base;

- creation of space gradiometers with an accuracy of $10^{-4} \div 10^{-5}$ Eotvos to prepare maps of remote land and sea areas. In the foreign space project GOCE [1], the achieved error in measuring the gradient was 0.001 Eotvos, in the GRACE project [2], the error is an order of magnitude less. At the same time, the use of data from these meters makes it possible to create maps only with a resolution of the order of $100 \mathrm{~km}$;

2) the problem of creating high-precision onboard sensors of the EGF. The accuracy of onboard sea and air gravimeters achieved to date is $0.5 \div 1.0 \mathrm{mGal}$, the accuracy of onboard gradiometers is $10 \div 100$ Eotvos. To create "seamless" gravimetric navigation systems, an increase in the accuracy of the listed onboard gravitational sensors is required, at least by 1 2 orders of magnitude.

\section{Problems of magnetometric navigation systems:}

1) the problem of improving the global model of the EMF. If we take into consideration the models of the EMF, then in terms of the number of expansion coefficients they are much inferior to the models of the EGF. The BGGM model [3] has the highest degree and order (1440), which includes the main geomagnetic field and the field of crustal anomalies. However, it should be noted that the existing global model of EMF anomalies EMAGv2 has a resolution of 2 arc minutes [4], which corresponds to a spatial resolution of about $3.5 \mathrm{~km}$. Increased spatial resolution is needed;

2) the problem of regularly updating the EMF model. An important feature of the EMF is its variability over time due to the instability of the solar parameters. Magnetic observatories and magnetovariational stations are used to account for variations in the parameters of the EMF. Due to the variability of the EMF, to refine its global model, additional measurements of its parameters are required once every 4-5 years. 
The park of the EMF parameters meters, in contrast to meters of the EGF, is quite extensive. There are magnetometers for measuring both the total value of the EMF (quantum, proton, etc.) and its components (fluxtage). The sensitivity of the existing magnetometers is at the level of picoTesla units (pT), the errors are less than $1 \mathrm{nT}$, and the measurement frequency is up to $1000 \mathrm{~Hz}$. Existing magnetometers can be used for land, sea, air and space measurements of magnetic field parameters.

\section{Features of navigation maps of the gravity field}

\subsection{Possibilities of using modern models of the EGF}

The simplest way to prepare navigational gravity charts is to use global gravity field models. At the same time, based on the results of modelling gravitational CENS, the required spatial resolution of the maps should not exceed hundreds of meters at present, and in the future - units to tens of meters.

There are a large number of models of the EGF of various degrees and orders. The most famous foreign models of the maximum degree and order of 2190 are EGM2008, EIGEN-6C4 and GECO [5]. These models provide a spatial resolution of about $18 \mathrm{~km}$, which is insufficient for navigation. In Russia, the most famous models of the EGF are PZ-90.11 [6] and GAO2012 [7], which have a degree and order of 360. These models have a spatial resolution of about $111 \mathrm{~km}$.

In FSUE "VNIIFTRI" in 2017, a gravitational field model was created, which is a fully normalized harmonic expansion coefficients of the geopotential in a series in spherical functions up to the degree and of the order of 720 (spatial resolution about $53 \mathrm{~km}$ ) [8]. The model parameters are obtained from joint processing of the original satellite and ground measurement data.

The values of geodetic parameters, the list and numerical characteristics of the parameters of the general terrestrial ellipsoid, used in the development of the model, correspond to the values of these parameters for the PZ-90.11 coordinate system.

The assessment of the accuracy of the gravitational field model was carried out by comparing the gravity anomalies calculated on the basis of the created model and the measured values of the gravity anomalies at three high-precision test sites in different parts of Russia: Central, Northwestern and Eastern. The total number of measurement points is 276. At the same time, the created model is also of little use for creating navigation maps, since has an unsatisfactory spatial resolution. Therefore, it is necessary to further search for methods to increase the spatial resolution (detail) of navigation maps.

One of them is based on the creation of high-resolution regional models of the EGF [9]. Another method is based on the use of additional information of the topography and density of the underlying rocks (see Section 5.2). One of the possible solutions is to search for pronounced anomalies of the gravity field, ensuring high accuracy of navigation, for example, in mountain gorges and in the buildings of reinforced concrete and brick structures (see Sections 5.4-5.5).

\subsection{The method of increasing regional gravity maps accuracy and spatial resolution}

The simplest solution to increase regional gravity maps spatial resolution are methods based on the interpolation of the measured gravity values. However, such methods have low accuracy, since significant errors in interpolation introduce uncertainties of the topography and rocks density ignorance at the distance between the original nodal points. 
The essence of the proposed method is illustrated in Fig. 3 [10]. Free fall acceleration $g$ at a point on the Earth's surface can be represented in the form [11]:

$$
g=\gamma_{0}+\delta g_{h}+\Delta g_{H}+\Delta g_{\Delta \rho},
$$

where $\gamma_{0}$ - is the normal Earth gravity field; $\delta g_{h}$ - is an anomalous addition due to the geodetic height of a point; $\Delta g_{H}$ - is an anomalous addition due to the influence of an intermediate layer of rocks enclosed between the surface of the Earth's ellipsoid and the physical surface of the Earth; $\Delta g_{\Delta \rho}$ - is an anomalous component of gravity caused by the influence of the inhomogeneity of the density of rocks below the surface of the ellipsoid.

The quantity $\gamma_{0}$ is determined by the well-known equation:

$$
\gamma_{0}=g_{e} \cdot\left(1+\beta \sin ^{2} \varphi-\beta_{1} \sin ^{2} 2 \varphi\right),
$$

where $g_{e}$ - gravity value at the equator; $\beta=\left(g_{p}-g_{e}\right) / g_{e} ; g_{p}$ - gravity value at the pole; $\varphi$ - point latitude; $\beta_{1}=\alpha \beta / 4+\alpha^{2} / 8, \alpha$ - common terrestrial ellipsoid compression.

Value $\delta g_{h}$ is determined by equation:

where $h$ - point geodetic height.

$$
\delta g_{h}=0,3086 \cdot h
$$

The abnormal addition due to the influence of the intermediate layer $\Delta g_{H}$ is generally determined by equation:

$$
\Delta g_{H}=\sum_{200 \text { ки }} \frac{G \rho_{i} \cdot v_{i}}{r_{i}^{2}},
$$

where $G$ - gravity constant; $\rho_{i}-$ unit cell density of rocks in the intermediate layer; $v_{i}-$ unit cell volume of the intermediate layer rocks; $r_{i}$ - distance to the unit cell of the rocks of the intermediate layer.

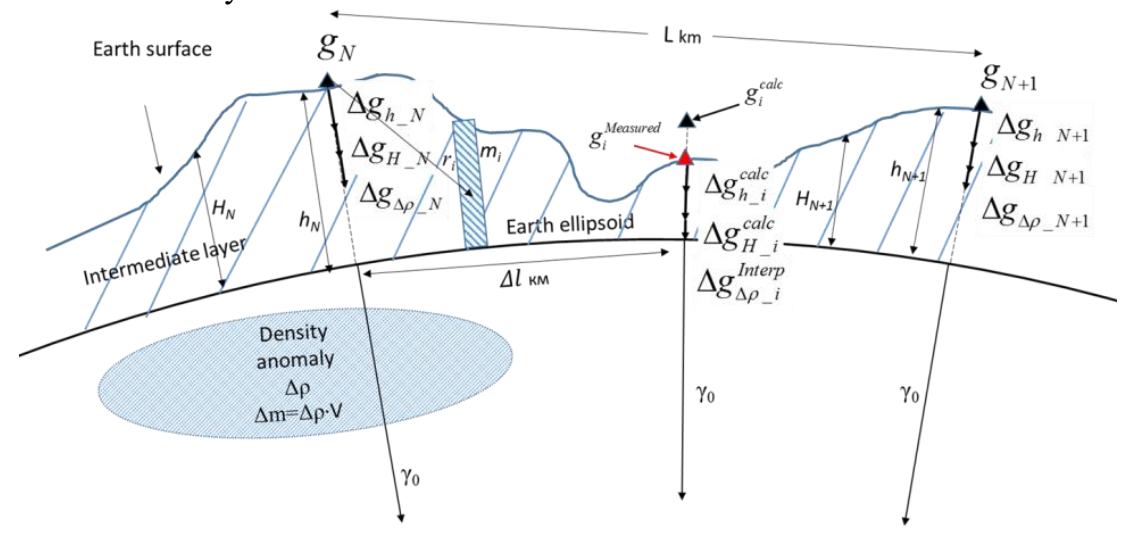

Fig. 3. The proposed method scheme

The abnormal addition $\Delta g_{\Delta \rho}$ is unknown, however, at the $i$-th point with known values of $g^{\text {Measured }}$ (taken from the map, or measured), this value can be calculated based on equation (2):

$$
\Delta g_{\Delta \rho_{-} i}^{\text {Calc }}=g_{i}^{\text {Measured }}-\left(\gamma_{0}+\delta g_{h}+\Delta g_{H}\right)_{i}^{\text {Calc }}
$$


where sum $\left(\gamma_{0}+\delta g_{h}+\Delta g_{H}\right)_{i}^{\text {Calc }}$ calculated by the above equations (2), (3), (4).

Unit $\Delta g_{H}$ can be calculated by equation (4) according to the known information about the terrain heights and the values of the density of rocks around the point $g^{\text {calc }}$. These data are freely available in the form of digital elevation models [12] and digital rock density models [13]. Information about the heights of the relief is presented in the form of a set of rectangular prisms; therefore, the component $\left(G \rho_{i} \cdot v_{i}\right) / r_{i}^{2}$ in equation (4) is calculated as follows:

$$
\begin{aligned}
& \frac{G \rho_{i} \cdot v_{i}}{r_{i}^{2}}=-G \rho_{i} \int_{\xi_{1}}^{\xi_{2}} \int_{\eta_{1}}^{\eta_{2}} \int_{\zeta_{1}}^{\zeta_{2}}[(\xi-x) \ln (\eta+R)+\eta \ln [(\xi-x)+R]+ \\
& \left.+\zeta \operatorname{arctg}\left(\frac{\eta}{\zeta}\right)-\zeta \operatorname{arctg} \frac{(\xi-x) \eta}{\zeta R}\right] d x d y d z,
\end{aligned}
$$

where $G$ - gravity constant; $\rho_{i}-$ unit cell density of rocks; $\xi_{1}$ and $\xi_{2}$ - coordinates of the corners of the prism along the $\mathrm{X}$ axis; $\eta_{1}$ and $\eta_{2}$ - coordinates of the corners of the prism along the $\mathrm{Y}$ axis; $\zeta_{1}$ and $\zeta_{2}$ - coordinates of the corners of the prism along the $\mathrm{Z}$ axis; $R=\sqrt{x^{2}+y^{2}+z^{2}}$, where $x, y$ and $z$-calculation point coordinates.

For two neighboring points $g_{N}$ and $g_{N+1}$, using formulas (2), (3), (6) and (5), the values $\Delta g_{\Delta \rho N}$ and $\Delta g_{\Delta \rho N+1}$ are determined, on the basis of which interpolation to the intermediate $i$-th point is carried out by the equation:

$$
\Delta g_{\Delta \rho_{-} i}^{\text {Interp }}=\Delta g_{\Delta \rho N}+\left(\Delta g_{\Delta \rho N+1}-\Delta g_{\Delta \rho N}\right) \Delta l / L
$$

Where $\Delta g_{\Delta \rho_{-} i}^{\text {Interp }}$ - interpolated value of gravity anomaly; $\Delta g_{\Delta \rho N}$ and $\Delta g_{\Delta \rho N+1}$ - calculated by equation (5) gravity anomaly at the extreme points of the interpolation profile; $L-$ the length of interpolation profile; $\Delta l$-interpolation interval.

As a result, the gravity value $g_{i}^{\text {calc }}$ at the intermediate $i$-th point is determined by the ratio:

$$
g_{i}^{\text {Calc }}=\left(\gamma_{0}+\delta g_{h}+\Delta g_{H}\right)_{i}^{\text {Calc }}+\Delta g_{\Delta \rho_{-} i}^{\text {Interp }}
$$

The estimation of the method accuracy was carried out on the example of the measuring profile in Moscow region. The profile consists of 31 measurement points with a distance of $\approx 5 \mathrm{~km}$ between them. Using the proposed method, the gravity values were interpolated to intermediate points with a distance of $30 \mathrm{~m}$ between them. At eight new randomly selected points, control measurements were carried out using the CG-5 Autograv gravimeter. The error of the proposed method is estimated from the difference $\sigma g=g_{i}^{\text {Calc }}-g_{i}^{\text {Measured }}$. The results of evaluating this difference showed that the modulus of $\sigma g$ values do not exceed $1.1 \mathrm{mGal}$. For comparison, the accuracy of classical method of linear interpolation was estimated on the same measuring. Calculations have shown that the values for the classical method reach $3.3 \mathrm{mGal}$, which is significantly higher than in the proposed method.

The proposed method is advisable to use to improve the accuracy and spatial density of existing regional gravity maps. 


\subsection{Examples of maps of anomalous values of the parameters of the EGF and EMF}

Examples of maps of parameters of anomalous EGF of hilly terrain, built on the basis of the EGF model of FSUE "VNIIFTRI" and anomalous EMF, built according to the EMAGv2 model, are shown in Fig. 4.

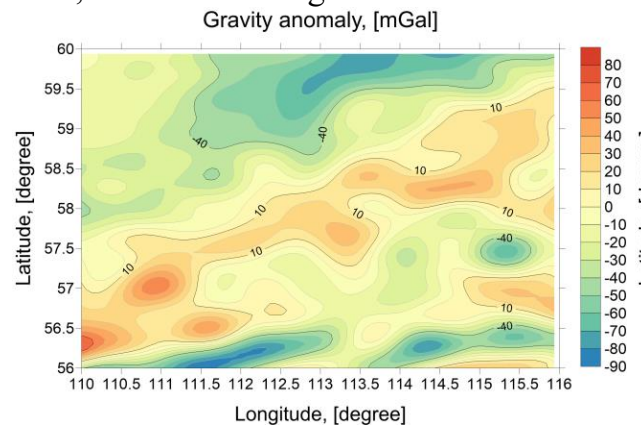

a)

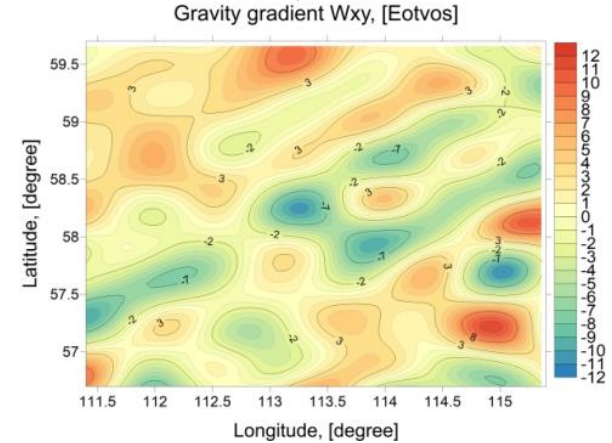

c)

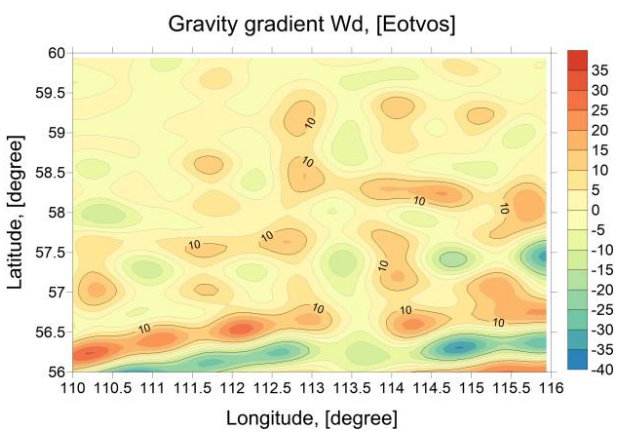

b)

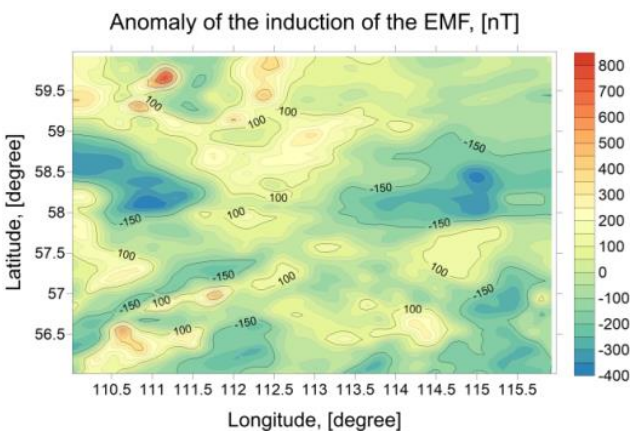

d)

Fig. 4. Examples of maps of anomalous values of the parameters of the EGF and EMF for the foothill relief

The given cards have the following characteristics:

a) anomaly of gravity: range of changes from -90 to $+80 \mathrm{mGal}$;

b) component of the EGF gradient $W_{\Delta}=W_{y y}-W_{x x}$ : range of changes from -40 to +35 Eotvos;

c) component of the EGF gradient $W_{x y}$ : range of changes from -12 to +12 Eotvos;

d) anomaly of EMF induction: the range of changes is from -400 to $+800 \mathrm{nT}$.

As can be seen from Fig. 4, the anomalous values of the parameters of the EGF and EMF in hilly terrain significantly exceed the errors of existing mobile gravimeters, gradiometers and magnetometers, and therefore are able to provide acceptable navigation accuracy.

\subsection{An example of a map of a highly informative anomalous Earth gravity field in a mountain gorge}

Examples of maps of parameters of a highly informative anomalous Earth gravity field in a mountain gorge, built on the basis of FSUE "VNIIFTRI" Earth gravity field model and the method presented in Section 5.2, are shown in Fig. 5. A sample of a mountain gorge was taken from a section of the Grand Canyon near Las Vegas, Nevada, USA. In the selected area, the depth of the gorge is $\approx 400 \mathrm{~m}$, width varies from 100 to $900 \mathrm{~m}$. Maps of gravity 
gradients $W_{\Delta}=W_{y y}-W_{x x}$ and $W_{x y}$ are prepared for two variants of movement of the navigation object: 1) flying at an altitude of $50 \mathrm{~m}$ above the channel of the gorge (Fig. 5a and 5c); 2) flying at an altitude of $300 \mathrm{~m}$ along the sides of the gorge (Fig. 5b and 5d).

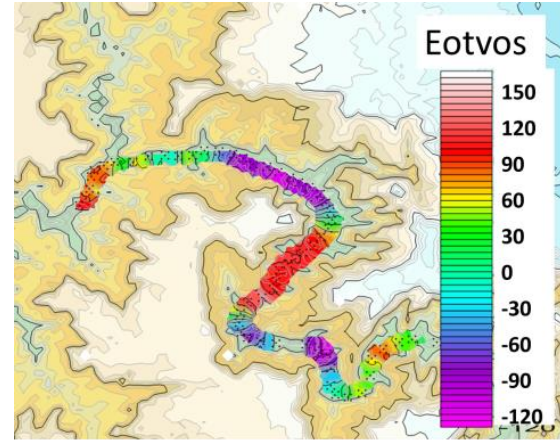

a)

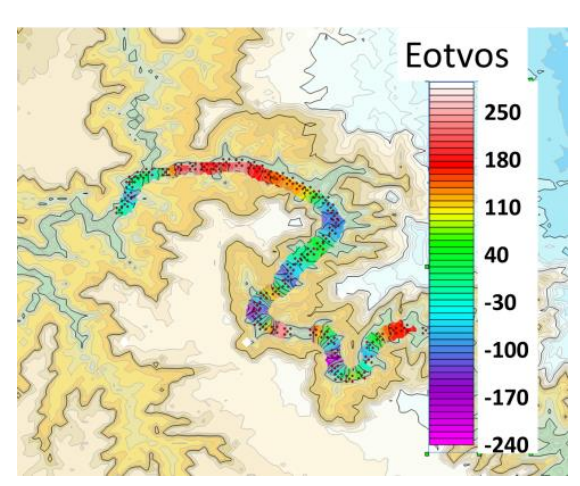

c)

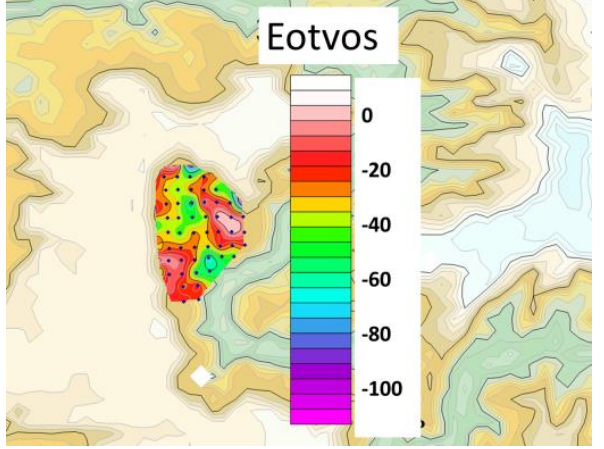

b)

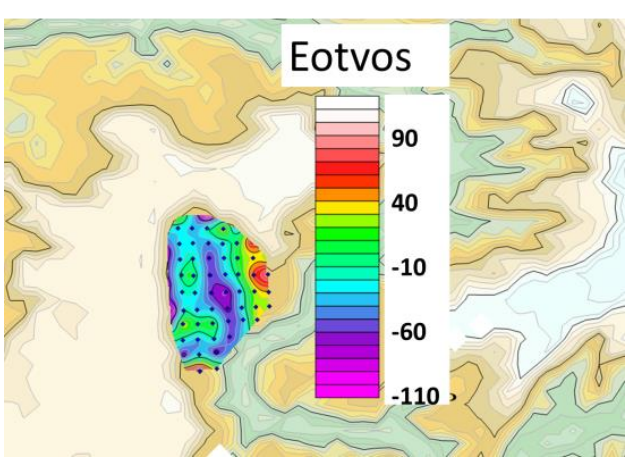

d)

Fig. 5. Examples of maps of highly informative anomalous Earth gravity field for a mountain gorge The given maps have the following characteristics:

a), b) component $W_{\Delta}=W_{y y}-W_{x x}$ of the Earth gravity field gradient: range of changes from -120 to +150 Eotvos;

c), d) component $W_{x y}$ of the Earth gravity field gradient: range of changes from -240 to +240 Eotvos;

The assessment of the navigation accuracy showed that the anomalous values of the Earth gravity field in the mountain gorge are capable of providing navigation accuracy of the order of hundreds of meters.

\subsection{Example of the gravity field anomaly map for buildings}

Examples of maps of parameters of a highly informative anomalous gravity field, constructed on the basis of information about the characteristics of the building and the method presented in Section 5.2, are presented in (Fig. 6). To model the spatial distribution of gravity values, gravitational gradients, $W_{\Delta}=W_{y y}-W_{x x}$ and $W_{x y}$ a building model of the following design was used:

- length, width, height: 18x10x9 m;

- number of floors: 3 ; 
- density of foundation and floor material: $2300 \mathrm{~kg} / \mathrm{m}^{3}$;

- material density of walls and partitions: $1800 \mathrm{~kg} / \mathrm{m}^{3}$.

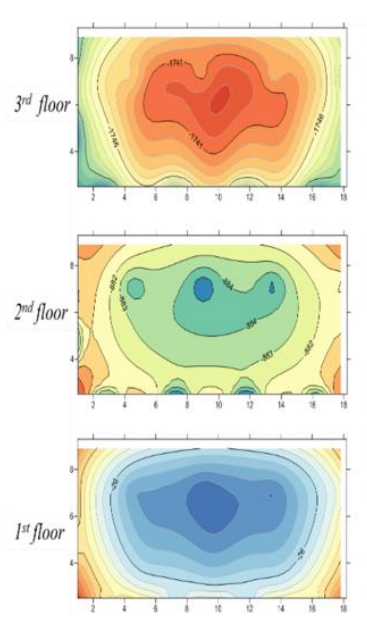

a)
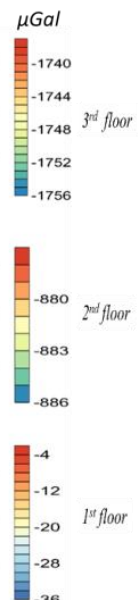

b)

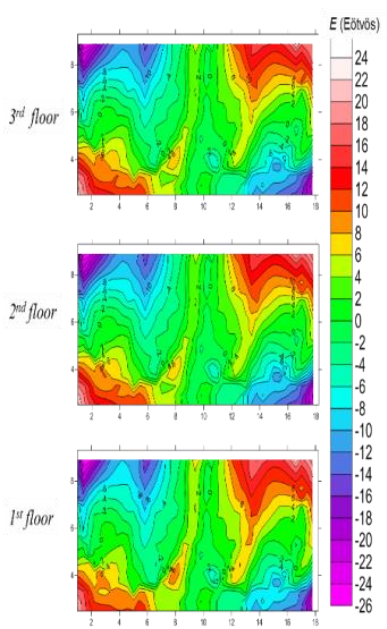

c)

Fig. 6. Examples of gravity field anomaly maps for buildings

The given maps have the following characteristics:

a) the change in gravity values between floors is $\approx 300 \mu \mathrm{Gal}$;

b) the change in the component of the gravitational gradient $W_{\Delta}=W_{y y}-W_{x x}$ along the floors of the building is from -190 to +110 Eotvos;

c) the change in the component of the gravitational gradient $W_{x y}$ along the floors of the building is from -22 to +20 Eotvos.

The assessment of the navigation accuracy showed that with the existing meter errors, the anomalous values of gravity field parameters in the building are capable of determining the position of the consumer with an error of no more than $1 \mathrm{~m} \mathrm{[14].}$

\section{New ground meters for preparing gravity maps}

\subsection{The existing park of ground-based gravimeters}

The existing fleet of ground-based free fall acceleration includes laser absolute ballistic gravimeters (AG) and high-precision relative gravimeters (RG).

AG are designed to measure the absolute value of free fall acceleration by measuring the free fall time of an optical corner reflector at a known height. The park of widely used AGs includes a number of foreign gravimeters from Micro-g LaCoste (FG-5X with an error of $2 \mu \mathrm{Gal}$, FG-L - $10 \mu \mathrm{Gal}, \mathrm{A}-10$ - $10 \mu \mathrm{Gal}$ ) [15], as well as Russian absolute gravimeters (GROT - $4 \mu \mathrm{Gal}$, GABL - $10 \mu \mathrm{Gal}$ ) [16]. The state primary standard of the unit of measurement of free fall acceleration in the Russian Federation has an error of $2 \mu \mathrm{Gal}$ [17]. The secondary free fall acceleration standard based on AG FG-L is located at FSUE "VNIIFTRI".

AG can be used to create a network of control points with absolute free fall acceleration values. The distances between them can be tens or hundreds of kilometers. A common disadvantage of all types of AGs is the significant required measurement time at one point, which is $20 \div 30$ hours.

To build a detailed map, RGs are used, which are designed to measure the increment of free fall acceleration relative to a known reference value. The main feature of $R G$ is high 
efficiency (no more than $20 \div 30$ minutes per point) and high measurement accuracy. Among foreign RGs, which are widely used to create navigational maps, one should mention the relocatable RG Scintrex CG-6 with an error of $5 \mu \mathrm{Gal}$ (Canada) [18] and mobile air RG GT-2a with an error of $0.5 \mathrm{mGal}$ (Canada) [19]. Among domestic mobile sea-based RGs, one should mention the Chekan-AM mobile gravimeter, the Shelf model, with a measurement error of less than $0.5 \mathrm{mGal}$ [20].

\subsection{Atomic interference absolute gravimeters}

Recently, in many countries of the world, the direction of creating absolute gravimeters and gradiometers has been widely developed, the principle of which is based on the interference of material waves (de Broglie waves) of ultracold atoms. The use of the wave properties of ultracold atoms makes it possible to implement atomic interferometry methods that are sensitive to the acceleration of gravity. Currently, the development of such devices in France [21], China [22], USA [23] and a number of other countries are known.

The measurement time with cold atoms gravimeters is about an order of magnitude shorter than in absolute ballistic gravimeters and is about 1 hour per point. At the same time, the measurement error is already on the order of $3 \div 5 \mu \mathrm{Gal}$, which is commensurate with the errors of absolute ballistic gravimeters. The prospective achievable error of cold atom gravimeters is about $10^{-3} \mu \mathrm{Gal}$.

The FSUE "VNIIFTRI" is creating a gravimeter on cold atoms based on the experience in the development of quantum frequency and time standards (FTS) of the "fountain" type [24].

\subsection{Digital zenith camera for determination deflection of vertical}

Since the last decades, with the advent of small-sized telescopes and highly sensitive astronomical cameras based on CCD and CMOS technologies, digital zenith cameras (DZC) for determination deflection of vertical (DOV) have become widespread, based on the comparison of astronomical and geodetic coordinates of the telescope location [25]. DZC are fully automated and allow to determine the current values of the DOV components $\xi, \eta$ in real time with an error of $0.1^{\prime \prime} \div 0.3^{\prime \prime}$ within a time interval of no more than 1 hour.

The FSUE "VNIIFTRI" has developed an DZC based on the use of a digital small-sized telescope, an optical CCD-matrix, as well as specially developed software [26, 27].

The main feature of the developed DZC, in comparison with existing domestic and foreign samples, is a new measurement method. In this method, in each series of measurements, the calibration coefficients of the DZC are estimated, which makes it possible to take into account their change between series of measurements. This eliminates the process of initial calibration of the device, which increases the efficiency and productivity of night measurements. At the same time, the requirements for ensuring the rigidity of the horizontal base are reduced, which opens up new possibilities for carrying out field measurements on unprepared sites.

The tests of the DZC were carried out in various geographical points of the North-West region of Russia (in the Novgorod, Moscow and Kaluga regions).

During the tests, which were carried out in May-August 2018, measurements were made over 14 observation nights. On each observation night, 10 measurements were performed with the duration of measurements at each point not exceeding 45 minutes. The standard deviation of the DOV components measurements was in the range of $0.07 " \div 0.25$ " (Fig. 7). 


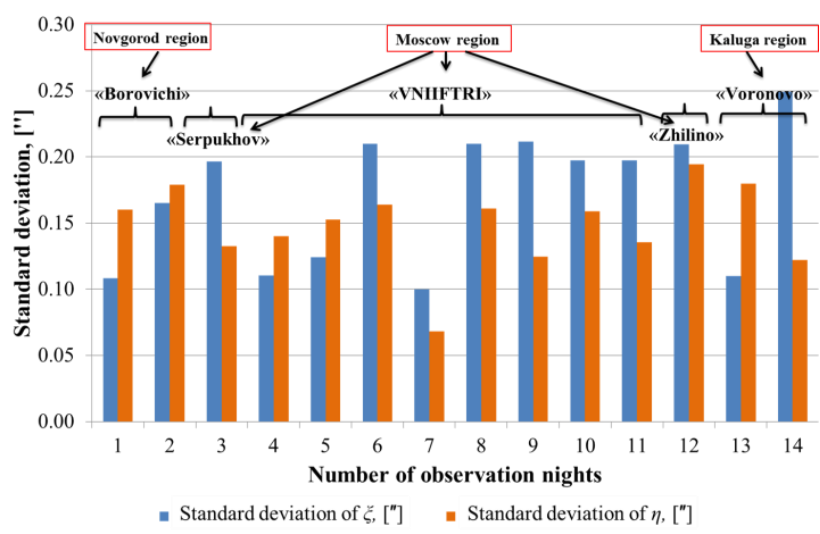

Fig. 7. Errors in determining the components of the DOV

\subsection{Modernization of the horizontal gravity gradiometer}

Horizontal gravity gradiometer E-60, the principle of operation of which is based on the use of a torsion balance, allows the determination of horizontal gradients of gravity [28]. The device weighs about $70 \mathrm{~kg}$. The device is controlled and readings are carried out manually. This requires a trained operator and significant measurement time. Increasing the accuracy and efficiency of the gradiometer measurements is possible with the use of modern optical measuring technologies and measurement processing methods. For example, in [29], when replacing the optical eyepiece E-60 with a digital camera and using a modern barcode rail, it was possible to reduce the error of the gradiometer from 2 to 0.2 Eotvos.

A prototype of a gravitational gradiometer with a capacitive measuring system has been developed at FSUE "VNIIFTRI" (Fig. 8a). A rotary platform is used to orient the prototype to a certain azimuth. This allows an increase in the amount of measurement information and measurements in any given azimuth using remote access. In addition, the prototype implements an automatic alignment system using linear actuators. Ultimately, this leads to a significant reduction in measurement time and complete automation of the measurement process.

To assess the sensitivity of the prototype, an experiment was performed using a perturbing weight of a cubic shape with a length of $10 \mathrm{~cm}$ and a weight of about $20 \mathrm{~kg}$. (Fig. 8b). The sensitivity of the first version of the device is estimated at $8 \div 10$ Eotvos. Currently, work is underway to increase the sensitivity.

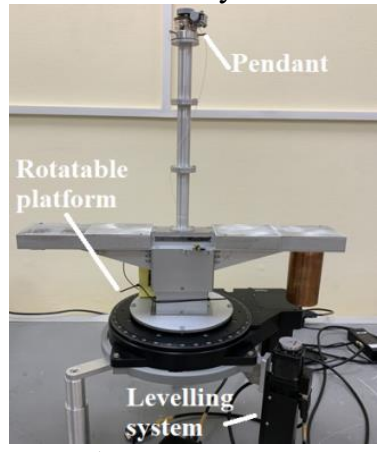

a)

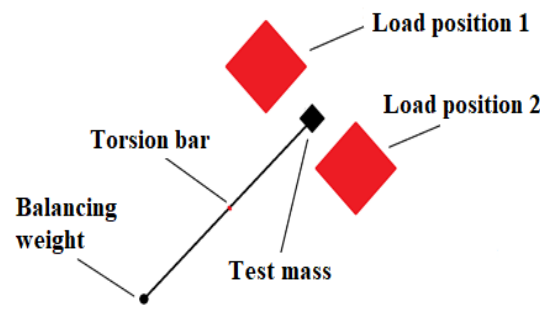

b)

Fig. 8. Gravity gradiometer: a) prototype; b) experiment scheme 


\subsection{Quantum level}

In 2015, at the Prague IAG conference, a special resolution was adopted "On the definition and implementation of the International Height Reference System (IHRS)" [30]. According to this resolution, the equipotential surface of the geoid should be taken as the reference surface when determining the height, and the height of any point on the Earth should be measured relative to it. It is proposed to determine these heights through the equivalent difference of gravitational potentials $\Delta C_{G}=\varphi_{0}-\varphi_{M}$, which is called the geopotential number. Here $\varphi_{0}=6,26368534 \cdot 10^{7} \mathrm{~m}^{2} / \mathrm{s}^{2}$ is the gravitational potential on the surface of the geoid, which is constant for any latitude and longitude; $\varphi_{m}-$ potential at the investigated point, the value of which is determined by the spatial coordinates of the point in the International Terrestrial Coordinate System ITRF.

On the basis of this resolution, since 2017, the International Association of Geodesy IAG has created the IHRS service, the main task of which is to establish a single highprecision global system of heights. IHRF Implementation Strategy Working Group established.

In the new system for determining heights, the measured physical quantity is the difference in gravitational potentials. According to the general theory of relativity, the physical effects directly related to the difference in gravitational potentials are the effects of gravitational frequency shift and gravitational deceleration (shift) of time. These effects are currently measured using highly stable FTS. In turn, the geopotential number in the first approximation is linearly related to the difference between the orthometric heights of the geoid and the point under study through the value of the gravitational acceleration.

For stationary ground-based atomic clocks, the formula for these effects is presented in the following approximate form [31, 32]:

$$
\frac{\Delta \tau_{G R}}{\tau}=\frac{\Delta f_{G R}}{f}=\frac{\Delta C_{m}}{c^{2}} \approx \frac{g_{m} \cdot \Delta H_{O R T}}{c^{2}},
$$

where $\Delta \tau_{G R}$ is the effect of the gravitational divergence of the time scales of the FTS on the time interval $\tau ; \frac{\Delta f_{G R}}{f}-$ gravitational displacement of the frequency of the master oscillator of a highly stable FTS; $g_{m}$ - absolute value of free fall acceleration; $\Delta C_{m}=\varphi_{0}-\varphi_{m}-$ geopotential number; $c$ is the speed of light; $\Delta H_{O R T}-$ the required difference in orthometric heights.

The International Global Geodetic System GGOS has established a requirement for measuring the geopotential number with an error of $10^{-2} \mathrm{~m}^{2} / \mathrm{s}^{2}$. As follows from the previous formula, in order to achieve such an accuracy, a TFS with a relative instability of $10^{-19}$ is required, and the error in measuring the difference in the orthometric heights of points on the Earth's surface is $1 \mathrm{~mm}$.

The set of tools necessary for measuring the geopotential number and the corresponding difference in orthometric heights includes two spaced FTS and a system for their synchronization. Such a complex can be called a quantum level [33]. Compared to a classical level, its error is weakly dependent on the distance between the studied points and, depending on the capabilities of the synchronization system, it can perform global measurements. Synchronization can be performed using GNSS, VLBI signals, as well as using fiber-optic communication lines (FOCL). The synchronization error for GNSS and VLBI is $0.1 \div 0.3 \mathrm{~ns}$. The achievable error of comparing two frequency frequencies using 
FOCL is $10^{-17} \div 10^{-19}$, which is shown both in foreign [34, 35] and in Russian experiments (Physical Institute named after P.N. Lebedev RAS, FSUE "VNIIFTRI").

At FSUE "VNIIFTRI", two models of a quantum level was created based on a relocatable quantum hydrogen clock with an instability of $1 \cdot 10^{-15}$ and $4 \cdot 10^{-15}$. On their basis, experiments were carried out to measure geopotential numbers between the State Standard of Time and Frequency, located at FSUE "VNIIFTRI" and points in the Caucasus (height difference $+1804 \mathrm{~m}$ ) [36], as well as in Nizhny Novgorod (height difference minus $65 \mathrm{~m}$ ) [37]. Synchronization on the route of movement of the relocated FTS and at the points of measurement was carried out using GLONASS signals. Experiments have confirmed the performance of both layouts.

In order to improve the accuracy of comparing the time scales of two FTS of a quantum level, a method of relativistic synchronization of FTS based on compensation of relativistic displacements of the time scale on the FTS movement path was proposed and tested by measuring the current coordinates and velocity using the GLONASS onboard navigation equipment [38]. With a path length of up to $1000 \mathrm{~km}$, the expected error of the method, determined by the capabilities of modern navigation equipment in differential mode, is a few picoseconds.

In addition to measuring the difference in orthometric heights, a quantum level opens up a number of other possibilities:

1. Improving the accuracy of transporting the time scale using mobile FTS based on relativistic synchronization. The method was experimentally confirmed when the FTS moved along the Moscow-Irkutsk highway over a distance of more than $5000 \mathrm{~km} \mathrm{[39].} \mathrm{The}$ time scale transmission error did not exceed $150 \mathrm{ps,} \mathrm{which} \mathrm{is} \mathrm{significantly} \mathrm{better} \mathrm{than} \mathrm{when}$ using other methods.

2. Creation of a distributed network of quantum levels for continuous monitoring of the difference in orthometric heights (geopotential numbers) between remote points. The scheme of such a monitoring system includes the Central FTS, regional FTS, mobile FTS and various synchronization systems between them. It is proposed to name the central FTS with the highest stability $\left(10^{-18} \div 10^{-19}\right)$ "Quantum tide gauge", by analogy with the Kronstadt tide gauge in the system of classical levels [33]. With respect to the quantum tide gauge, changes in orthometric heights are counted across the entire network of quantum levels. The "Quantum tide gauge" network will allow creating a single altitude base of the country with the same altitude determination error at any point: on the islands, in the Arctic region, etc. The reference mismatch of the time scales of terrestrial TFS can be performed using FOCL, the method of relativistic synchronization, the Duplex synchronization complex, quantum-optical system (QOS) and using the GLONASS spacecraft. The conceptual diagram of the "Quantum tide gauge" network is shown in Fig. 9.

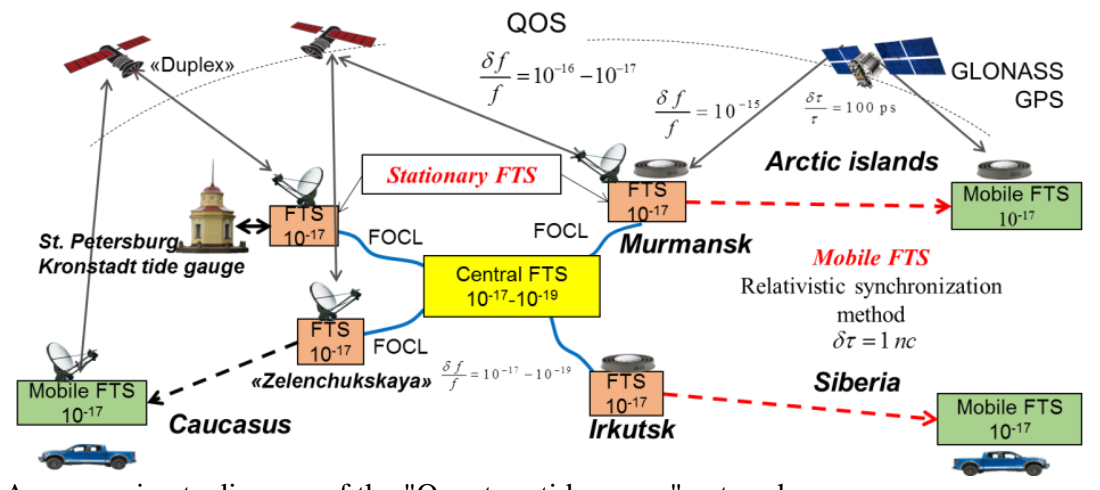

Fig. 9. An approximate diagram of the "Quantum tide gauge" network 
Abroad, in particular, in Europe, ground-based networks for monitoring geopotential numbers are already being created on the basis of FTS with an instability of $10^{-17} \div 10^{-18}$ using synchronization systems based on fiber-optic lines. In the GGOS system, according to a similar scheme, it is planned to create the IHRF International Height Reference System.

In addition to the main task, the "Quantum tide gauge" network will allow monitoring the current values of the components of the deviations of the plumb line:

$$
\xi=-\frac{1}{\gamma_{c p}} \cdot \frac{\left(\varphi_{2}-\varphi_{1}\right)_{x}}{x_{2}-x_{1}}, \quad \eta=-\frac{1}{\gamma_{c p}} \cdot \frac{\left(\varphi_{2}-\varphi_{1}\right)_{y}}{y_{2}-y_{1}},
$$

where $\xi, \eta$ - the components of the DOV in latitude and longitude; $x_{2}-x_{1}, y_{2}-y_{1}-$ distance between measurement points along the $O X$ and $O Y$ axes; $\varphi_{2}-\varphi_{1}$ - the difference in gravitational potentials between the measurement points; $\gamma_{c p}-$ the average value of free fall acceleration between the points of measurements.

\section{Space meters of gravitational field parameters}

\subsection{Foreign space gravimetric projects}

Space gradiometer. To date, the highest level of accuracy in measuring the gradient in space has been achieved on a gradiometer made in the form of 3 orthogonal pairs of triaxial spherical microaccelerometers placed on the geodetic satellite of the European Space Agency GOCE [2]. The project started in 2009 and completed in 2013. In this project, 6 highly sensitive ball-in-a-sphere microaccelerometers are placed around the satellite's center of mass and, due to their design, allow measurements along $6 \times 3=18$ axes. The distance between accelerometers along each axis is $0.5 \mathrm{~m}$. The error of each accelerometer along all three measuring axes is about $2 \cdot 10^{-12} \mathrm{~m} / \mathrm{s}^{2}$, which is close to the thermodynamic limit of mechanical accelerometers. The measurement error in such a gradiometer is approximately $(2 \div 3) \cdot 10^{-3}$ Eotvos, or about $10^{-6}$ relative to the magnitude of the Earth's gradient. Based on the results of 4-year measurements, the global EGF model was refined.

Judging by the latest publications, new, more advanced versions of the GOCE project are not planned abroad yet. This is because gradiometers of this type have approached their accuracy limit.

Satellite-to-satellite meters. Measurements along the "satellite-to-satellite" line in order to clarify the parameters of the gravitational field were first implemented by NASA in the low-orbit system GRACE (2002-2012) [1]. This system consists of two satellites moving in tandem in the same low orbit at a distance of $220 \mathrm{~km}$ from each other. The current mutual distance was monitored using a specially created high-precision radio-technical rangefinder with a measurement error of $1 \mu \mathrm{m}$, which ensured a sufficiently high accuracy in determining the anomalies of the gravitational field. The absolute error in measuring the gradient is $10^{-4}$ Eotvos.

Currently, a new version of this GRACE-FO project is being implemented (since 2018) [40]. This project uses high-precision laser measurements of the relative motion of two satellites with an error of $30 \div 50 \mathrm{~nm}$. This will make it possible to obtain a higher accuracy of measurements of the parameters of the EGF. A similar project is being implemented in China. In addition, the concept of the GRACE-2 system, based on the use of networked multi-satellite systems, is being developed.

Space radio altimeters (altimeters). The world leader and monopolist in the field of space altimeters creation is the foreign company Thales Alenia Space. For three decades, it 
has been producing onboard altimeters for monitoring the surface of the world's oceans, ice cover, hydrology, as well as for detecting anomalies of the Earth's gravitational field and sea surface slopes. The achieved level of accuracy in measuring slopes, judging by the advertisement, is $10^{-6}$ radians $(1 \mathrm{~cm}$ per $10 \mathrm{~km})$. The range of the company's products includes standard altimeters, altimeters based on synthetic aperture radar, as well as altimeters in interferometer mode.

The idea of using GNSS signals for bistatic radar was proposed by one of the authors in the 90 s of the last century [41]. At present, space bistatic radio altimeters based on GPS and GALILEO GNSS signals are being actively studied abroad. Successful space experiments with such a device were performed by SSTL (Great Britain) [42]. At the University of Birmingham (Great Britain), experiments are being carried out with a radar of this type with its placement on board a helicopter [43]. A similar device was installed on board the CHAMP geodetic satellite; it is supposed to be used in the ACES space experiment; Germany is planning the GEROS experiment on the European segment of the International Space Station [44]. In the United States, the first stage (8 satellites) of the multi-satellite (24 satellites) bi-static radar system CYGNSS using GPS signals for radio backlight was implemented [45]. The objectives of the experiments are observation of the ocean surface, including the survey of its height profile coinciding with the geoid surface.

In 2011, an operating aviation radar was created in Russia on the reflected signals of GNSS GLONASS [46], radio images of real surface objects were obtained simultaneously from several angles from a height of about a kilometer. Flight altitude was measured at the same time. The radar can be used for high-precision measurement of the ocean surface elevation profile and, accordingly, the geoid elevation profile in the mode of direct measurement of the BT signal phase, as well as in the radio interferometer mode.

Space laser gradiometer. Abroad, within the framework of the LISA PATHFINDER project, created in the interests of detecting gravitational waves, a space experiment was carried out in 2016 to accurately measure the relative motion of two free masses inside the spacecraft [47]. A laser interferometer of a special design was used for measurements. The measurement error was $10^{-15} \mathrm{~m}$. This experiment is the basis for the creation of a low-orbit space laser gradiometer with a precision that is several orders higher than the accuracy of the GOCE gradiometer.

Space meter of gravitational potential. The European Space Agency plans to implement the ACES space project in 2019 [48], one of the goals of which is to measure the current gravitational potential between low-orbit satellites and ground-based radio and laser stations. For this purpose, the frequency and time standard of the fountain type PHARAO with an instability of $10^{-16} \div 10^{-17}$ is placed on board the spacecraft. The implementation of the project will allow linking the high-altitude foundations of all continents into a single system with an error of less than $1 \mathrm{~m}$.

\subsection{Russian developments of a bistatic radio altimeter based on GNSS signals}

Determination of the geoid profile is one of the main tasks of geodesy, gravimetry and oceanography. The surface of the geoid coincides with the surface of the World Ocean in the absence of disturbing forces such as wind, ocean currents, tides and conditionally continues under the continents. When describing a geoid, one resorts to the concept of a geoid height - the elevation of a geoid over an ellipsoid.

The principle of operation of the bistatic altimetry system based on GNSS signals is based on the reception of direct and reflected signals from GNSS navigation spacecraft (NSA). Direct signals are received by a weakly directional antenna directed towards the "zenith" (Fig. 10). Signals reflected from the ocean surface in the vicinity of the point of 
specular reflection are received by an antenna directed to the nadir. To solve the problem of determining the height to the reflecting surface, it is necessary to determine the difference between the propagation time of the direct and reflected signals, the coordinates and velocities of the receiver and the satellite. In addition, it is necessary to know the received signal strength, the model of the reflecting surface, tropospheric and ionospheric delays.

The advantage of a bistatic space radio altimeter based on GNSS signals is a large number of simultaneous measurements of the height above the water area. When using the signals of $30 \div 40$ visible satellites of the GLONASS, GPS, GALILEO, BaiDou, INSS systems, independent measurements can be performed on the same number of tracks at the same time. In a classic mono radio altimeter, measurements are taken along one track.

As noted, in 2011, an operating aviation radar based on reflected signals from GNSS GLONASS was created in the Russian Federation [46].

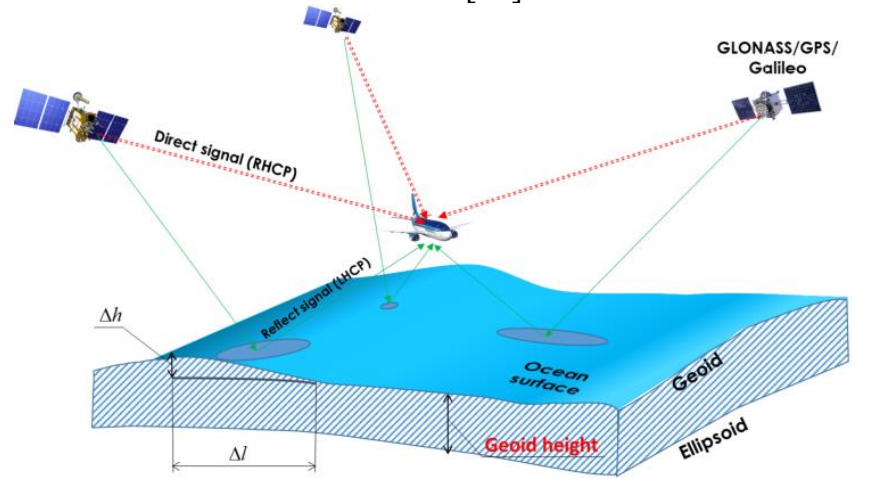

Fig. 10. To an explanation of the principle of operation of a bistatic radio altimeter based on reflected GNSS signals

The basic equation of the bistatic altimetry system for determining the height of the geoid in a simplified form can be written as follows:

$$
H_{g}=H_{e}-\frac{\rho_{r}-\rho_{d}}{2 \cdot \sin \theta},
$$

where $H_{g}$ - the height of the geoid; $H_{e}$ - geodetic height; $\theta$ - angle of incidence / reflection of the signal; $\rho_{r}$ and $\rho_{d}$ - distances traveled by reflected and direct signals.

This method, based on the increments of the geoid heights, also makes it possible to determine the values of the DOV components:

$$
\varepsilon_{\xi, \eta}=\frac{\Delta h_{(x, y)}}{\Delta l_{(x, y)}},
$$

where $\varepsilon_{(\xi, \eta)}$ - DOV component in latitude or longitude; $\Delta h_{(x, y)}$ - increment in height between two points along the OX or OY axis; $\Delta l_{(x, y)}$ - distance between two points along the OX or OY axis.

There are several methods of bistatic GNSS radio altimetry for determining the height of the geoid: code, interference, phase method and method of fixing the signal-to-noise ratio ( $\mathrm{S} / \mathrm{N}$ method).

To test the code method and the S/N method of bistatic GNSS altimetry, a number of experimental measurements of the height from a bridge over the water surface of the reservoir were carried out at FSUE VNIIFTRI [49]. Receiving antennas were located on the bridge at point 1 (Fig. 11). The direct signal was received by the antenna directed "towards the zenith", the reflected signals - by the antenna "towards the nadir". The true height above the water was measured using a laser rangefinder. 


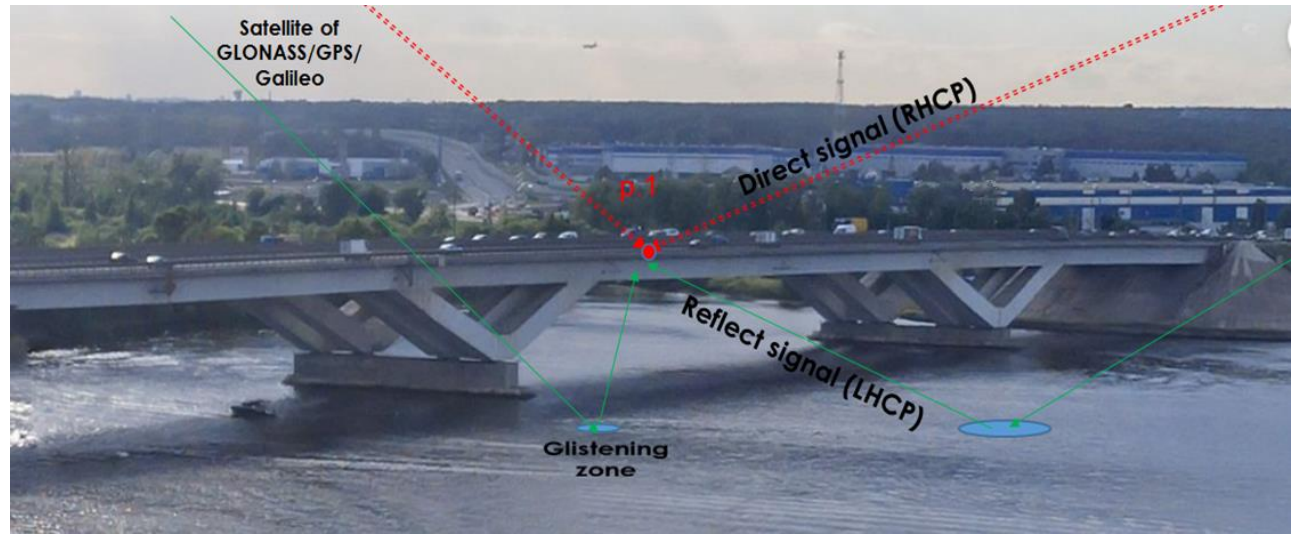

Fig. 11. Place of the experiment: p.1 - the location of the receiving antennas

Measurement results:

1) the difference between the true height and the height measured using the code method (static error) was $0.05 \mathrm{~m}$;

2) the difference in the same heights, measured on the basis of the $\mathrm{S} / \mathrm{N}$ method, is 0.01 $\mathrm{m}$.

Providing the conditions for the necessary excitement of the reflecting surface and the required elevation angles of the satellite is not always a feasible task in field measurements. Therefore, to test the phase method for determining the height above the reflecting surface, an anechoic shielded chamber (Fig. 12) was chosen, equipped with a GNSS simulator and wall-mounted navigation signal emitters.

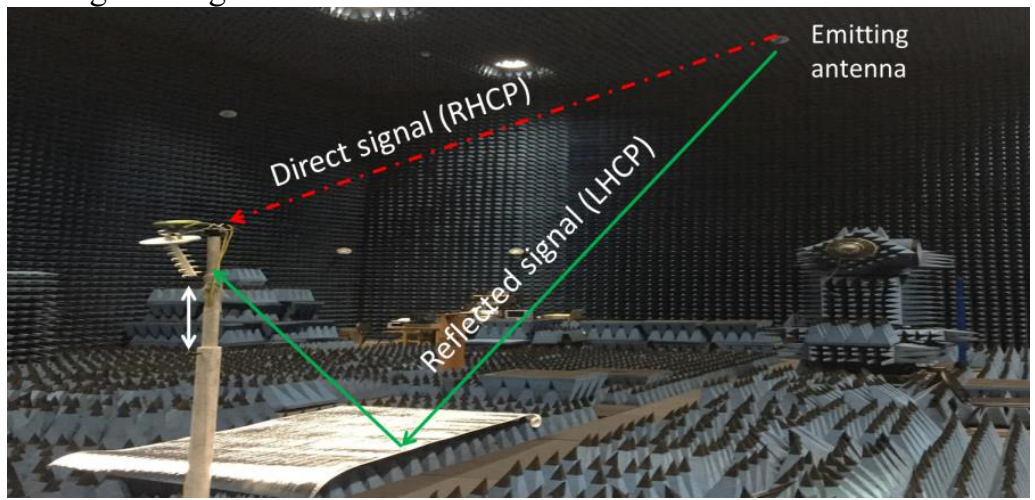

Fig. 12. The experimental scheme in an anechoic chamber

Fig. 13 shows the results of testing the phase method with a smooth change in the height of the receiving antenna located on a movable mast. It can be seen that even with a rapid change in altitude, starting from the 300th second ( $40 \mathrm{~cm}$ in 30 seconds), phase disruptions did not occur. The root mean square error of phase measurements did not exceed $2 \mathrm{~mm}$. 


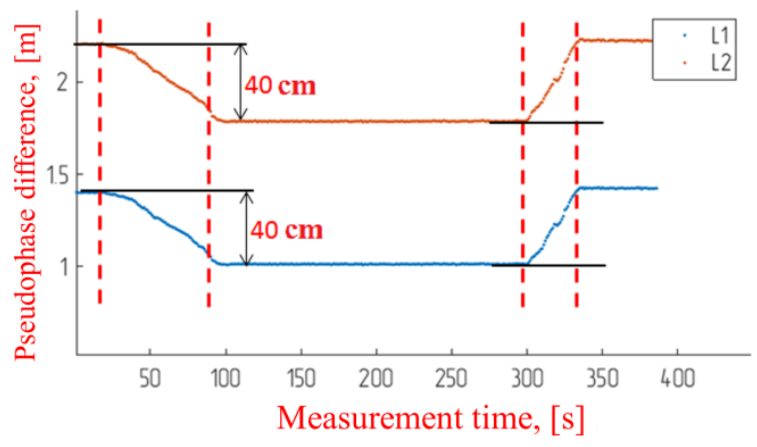

Fig. 13. Experimental results when changing the height of the mast: up to $100 \mathrm{~s}$, lowering the antennas down, from 300 to $330 \mathrm{~s}$, raising the antennas up

Methods of bistatic radio altimetry based on reflected GNSS signals are suitable for constructing geoid height profiles in the waters of the World Ocean. With a large number of simultaneously received signals from navigation spacecraft, one onboard GNSS-radio altimetry system is capable of giving much more measurement information in one pass of the spacecraft than an active altimeter. When using the phase method of measuring the profile of the geoid height, it is possible to achieve the centimeter level of accuracy.

\subsection{Space laser gradiometer}

As noted above, in the GRACE space mission, to measure active accelerations caused by the residual atmosphere, pressure from the Sun, etc., accelerometers of very high accuracy are placed on both satellites: their error should not exceed $10^{-11} \div 10^{-12} \mathrm{~m} / \mathrm{s}^{2}$. This is a very complex devices, which greatly complicate the onboard satellite equipment.

To exclude the installation of ultra-precise accelerometers on board a gravimetric spacecraft, a space gradiometer on free masses (FM) moving inside the spacecraft is proposed. [50, 51]. The second gradient of the gravity potential on board the spacecraft is calculated by measuring the difference in the gravitational forces acting on the FM, which move along one axis. The difference in the acting gravitational forces is calculated from the relative motion of the $\mathrm{SM}$, the parameters of which are measured using a laser interferometer (Fig. 14). To increase the effective distance between free masses, it is possible to use several pairs of reflecting mirrors, Fabry-Perot resonators, and optical fibers. The main advantage of the proposed gradiometer is the possibility of placing it on board one spacecraft without the need for an onboard high-precision accelerometer.

To assess the main parameters of the gradiometer, a software-mathematical model was developed. Modelling was carried out with the following initial data: satellite flight altitude $-250 \mathrm{~km}$; discreteness of reports $-1 \div 10 \mathrm{~s}$; airborne laser interferometer error $-0.01 \cdot 10^{-9}$ $\mathrm{m}$; distance between free masses $-1 \mathrm{~m}$. Based on the simulation results, the achievable sensitivity of the device was established: $0.0001 \div 0.00001$ Eotvos. 


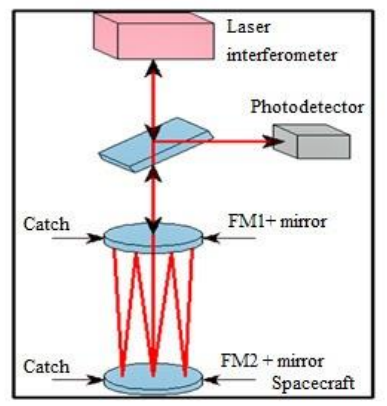

Fig. 14. The principle of operation of the laser gradiometer

Currently, the development of a ground-based model of a space laser gradiometer is being carried out. In this case, the results obtained when creating a transportable laser interferometer according to the Michelson interferometer scheme will be used.

\subsection{Promising directions of gravimetric research}

\subsubsection{The concept of "Relativistic Geodesy"}

Relativistic geodesy is a branch of higher geodesy that studies the influence of gravitational fields on the accuracy of space-time geodetic measurements. Without her conclusions, it is impossible to implement the strict requirements of the GGOS project. This direction of geodesy is rapidly developing both in Russia and abroad [for example, 33, 52].

The main task of relativistic geodesy is to establish the dependence of the measured geodetic length, time intervals, and frequency of FTS on the parameters of the EGF and the speed of movement on Earth and in near-Earth space.

Studies have shown that many factors affect the length of the measuring instruments, the frequency and the time scale of the FTS in the earth's rotating ITRS frame [33]. Below is the calculated level of influence of various relativistic and gravitational factors of nearEarth space on changes in length, frequency and time in relative values: $\frac{\Delta l_{G R}}{L}=\frac{\Delta \tau_{G R}}{\tau}=\frac{\Delta f_{G R}}{f}:$

- the difference between the gravitational potentials of the Earth at the points where the measuring atomic clock is located: at level $10^{-9}-10^{-10}$;

- speed difference between space and ground atomic clocks: at level $10^{-9} \div 10^{-10}$;

- difference in centrifugal potential at the points of the watch: about $10^{-12}$;

- irregularity of the Earth's rotation, which includes deviation of angular velocity, nutation, precession, and wobble of the pole line: $10^{-16} \div 10^{-17}$;

- tidal potential change in spacecraft orbit: $10^{-14}$;

- solar pressure: $10^{-15}$;

- tidal potential change on earth: $10^{-15}$;

- change in potential in orbit due to zonal harmonics of the EGF: $10^{-13}$.

The directions of development of relativistic geodesy are:

- Development of the relativistic theory of the interaction of FTS and electromagnetic waves in the atmosphere with the EGF in the Earth's rotating frame of reference ITRS at high velocities of the meter.

- Improving the accuracy of measurements of radio and optical geodetic, navigational aids, as well as means of synchronization of the FTS due to the correct consideration of the influence of relativistic phenomena in the EGF. 
- Measurement of relativistic effects (RE) on the Earth and in space in order to determine the characteristics of the EGF and geodetic parameters of the Earth's figure. To date, the relative error in the measurement of relativistic effects in GNSS, for example, GALILEO with airborne FTS with a relative instability of $10^{-14}$, has reached a value of $\frac{\delta(R E)}{(R E)}=10^{-5}[53,54]$. When using onboard optical frequency standards with frequency instability up to $10^{-17} \div 10^{-18}$, the relative measurement error of relativistic effects in space geodetic radio lines will increase to $10^{-8} \div 10^{-9}$, which corresponds to the best samples of geodetic instruments.

One of the practical outputs of relativistic geodesy (sometimes this direction of geodesy is called "Chronogeodesy") is to create a distributed network "Quantum tide gauge". It can be considered as an element of the IHRS global system of uniform heights being created.

\subsubsection{Relativistic characteristics of the near-earth "gravitational medium"}

The peculiarity of ground-space measuring radio lines for geodesy and navigation is that they run in the Earth's gravitational field, crossing the atmosphere. At the same time, they are considered from the terrestrial reference system ITRS (International Terrestrial Reference System), rigidly connected with the rotating Earth. In this system, due to the principle of equivalence in the general theory of relativity, an additional gravitational field (field of inertia forces) induced by rotation appears in near-earth space, the action of which on an electromagnetic wave is similar to the action of the true gravitational field of the Earth. Therefore, the task was to determine the relativistic refractive properties of nearEarth space, including the atmosphere, and relativistic additions to the parameters of radio wave propagation. The solution of the problem was carried out on the basis of the methods of the general theory of relativity [55]. In this case, Maxwell's tensor equations and generalized tensor material relations were used, which carry information about the atmosphere, gravitational field and rotation of the reference frame. For an electromagnetic wave in a moving isotropic conducting medium, the wave equation and the corresponding dispersion relation are obtained, the solution of which is the expression for the generalized refractive index of the near-Earth space [56, 57]:

$$
n * *=1+\Delta n_{a}+\left\{\frac{2 \varphi}{c^{2}}+\frac{\left[\mathbf{\Omega}_{\mathbf{0}} \mathbf{R}\right]^{2}}{2 c^{2}}+\frac{1}{2 c^{2}}\left(\left[\mathbf{\Omega}_{\mathbf{0}} \mathbf{R}\right] \mathbf{e}\right)^{2}\right\}+\frac{1}{c}\left[\mathbf{\Omega}_{\mathbf{0}} \mathbf{R}\right] \mathbf{e}
$$

where $\Delta n_{a}=n_{a}-1, n_{a}$ is the refractive index of the atmosphere; $\varphi$ - gravitational potential of the GPP; $\Omega_{0}$ - angular velocity of the ITRS system rotating with the Earth; $\boldsymbol{R}$ - the current radius vector of the radio wave; $\boldsymbol{e}$ - radio wave vector; $c$ - the speed of light.

In the plane of the Earth's equator, the value of the refractive index of this "gravitational medium" varies from $1.55 \cdot 10^{-6}$ on the Earth's surface to $10^{-5}$ in the geostationary orbit, which is commensurate with the characteristics of the ionosphere. At the same time, its influence on the propagation parameters is much higher, since this medium extends from the Earth to the geostationary orbit.

Another spatial characteristic of the "gravitational" near-earth medium is the coefficient of gravitational frequency conversion, or frequency activity:

$$
F=1+\frac{\varphi_{2}-\varphi_{1}}{c^{2}}+\frac{1}{2 c^{2}}\left(\left[\mathbf{\Omega}_{\mathbf{0}} \mathbf{R}_{\mathbf{2}}\right]^{2}-\left[\mathbf{\Omega}_{\mathbf{0}} \mathbf{R}_{1}\right]^{2}\right),
$$

where $\varphi$ is the gravitational potential of the EGF; indices 1 and 2 refer to the start and end of the propagation path, respectively. 
The coefficient $F$ determines the frequency-transforming properties of the near-earth "gravitational medium" in the rotating ITRS reference frame. It does not depend on the properties of the atmosphere and the speed of movement of the source and receiver of the radio wave and is determined only by the difference in gravitational potentials at the ends of the propagation path.

In addition, the "gravitational near-Earth medium" can be characterized by the index of relativistic refraction, as well as the index of the relativistic polarization of an electromagnetic wave [33].

In general, the near-earth "gravitational environment", from the point of view of radio wave propagation, has the following properties: 1) anisotropy, which leads to different delays of the counter rays; 2) inhomogeneity, which leads to refraction of radio and laser beams; 3) gravitational frequency conversion of radio and laser beams; 4) rotation of the plane of polarization of radio and laser beams; 5) commensurability of properties with the real ionosphere.

The proposed approach greatly simplifies the calculation of the parameters of radio waves and laser beams in high-precision space geodetic systems.

\subsubsection{Laser gravitational-wave antennas as an «engine» for ground and space gravimetry}

Currently, scientific projects are being developed in the world to create gravitational-wave antennas (GWA) on the Earth's surface and in space. The main task of the GWA is to register the movement of mechanical systems due to the effect of space curvature during the passage of a gravitational wave.

The most developed are ground-based laser interferometric detectors of gravitational waves: american gravity antenna LIGO [58]; antenna VIRGO, Pisa, Italy [59]; antenna GEO600, Hanover, Germany [60]; antenna KAGRA, Tokyo, Japan [61]. In 2015, two LIGO GWA laser detectors recorded gravitational waves for the first time. The wave originated from the merger of two black holes at a distance of 1.3 billion light-years away from Earth [58].

The most important technical characteristic of LIGO laser interferometers is the extremely high achieved sensitivity to changes in the distance between the mirrors. It is $10^{-}$ $18 \mathrm{~m}$, which is about 5-6 orders of magnitude more accurate than most of the widely used ground-based laser interferometers. This opens up new opportunities for their improvement. Therefore, the achievements in the creation of GWA can be considered as an «engine» for development of laser interferometers for both scientific and applied purposes.

In recent years, space projects of GWA have been actively developing, which represent a cluster of three or more spacecraft, each of which has two test masses. The mirrors of the laser interferometric intersatellite rangefinder are fixed on these masses. [61]. The most famous projects of such GWA are: LISA [62], DECIGO [64], TianQin [65], LAGRANGE [66], GEOGRAWI [67].

FSUE "VNIIFTRI" has developed a project for a domestic space GWA placed on the orbit of the GLONASS system: «SOIGA» (Space Optical Interferometric Gravitation Antenna) [68]. The antenna includes 4 inter-satellite laser interferometers in each orbit, while the effective length of the interferometer arm is 144 thousand $\mathrm{km}$. The length of the detected gravitational waves is in the range of $0.01 \div 10 \mathrm{~Hz}$. When creating SOIGA, it is supposed to use the existing developments for the creation of elements of GLONASS satellites and the accumulated experience in their launch and maintenance. Ultimately, this leads to a decrease in the cost of creating a GWA.

The most important practical application of the experience in the creation of groundbased GWA and the design of space GWA is the creation of promising high-precision 
space gravity gradiometers. It is important to note that along this path the gravimetric projects of all countries-developers of known GNSS are being developed: GPS, GALILEO, BaiDou, IRNSS, QZSS (Fig. 15). There is a hope that such developments in the future will provide high accuracy in the creation of global navigation-gravimetric maps in the interests of navigation through the gas processing plant.

\begin{tabular}{|c|c|c|c|c|}
\hline $\begin{array}{l}\text { Country- } \\
\text { developer }\end{array}$ & $\begin{array}{l}\text { Name } \\
\text { GNSS }\end{array}$ & $\begin{array}{c}\text { Name } \\
\text { detector }\end{array}$ & $\begin{array}{c}\text { The existing } \\
\text { space } \\
\text { gradiometer }\end{array}$ & $\begin{array}{c}\text { Perspective space } \\
\text { gradiometer }\end{array}$ \\
\hline USA & GPS & $\begin{array}{l}\text { LIGO } \\
\text { LISA }\end{array}$ & $\begin{array}{c}\text { GRACE } \\
\text { GRACE-FO }\end{array}$ & $\begin{array}{c}\text { Onboard laser GG } \\
\text { based on the } \\
\text { PLISA principle }\end{array}$ \\
\hline $\begin{array}{r}\text { European } \\
\text { Union }\end{array}$ & Galileo & $\begin{array}{l}\text { VIRGO, GEO-600 } \\
\text { EXPLORER, } \\
\text { AURIGA }\end{array}$ & GOCE & - \\
\hline CHINA & Beidou & TAIJI & - & TIANQIN \\
\hline INDIA & IRNSS & INDIGO & - & - \\
\hline JAPAN & QZSS & $\begin{array}{l}\text { TAMA300 } \\
\text { KAGRA, } \\
\text { DECIGO }\end{array}$ & - & - \\
\hline SSIA & ГЛОНАСС & - & - & ГЕО-ИК 3 \\
\hline
\end{tabular}

Fig. 15. GNSS and gravitational wave antennas developers

\section{Conclusion}

Thus, autonomous navigation based on the use of the parameters of the Earth's gravitational and magnetic fields is one of the most effective directions for the development of assisting GNSS technologies in difficult conditions. The navigation error for the EGF and EMF is currently hundreds of meters. However, in the future, it is possible to reduce the error to units of meters, provided that the following tasks are solved:

- refinement of global planetary models of the EGF and EMF;

- creation of a park of high-precision on-board gravimeters and gradiometers;

- creation of more accurate space laser gradiometers;

- improving the accuracy of quantum levels based on highly stable standards of frequency and time and high-precision synchronization to ensure a high-precision highaltitude base of large areas (the "Quantum tide gauge" network);

- creation of gravimetric instruments of a new type based on GNSS signals: bistatic multi-position radio altimeters;

- development of methods for operational preparation of navigation-gravimetric and navigation-magnetometric maps using GNSS;

- development of methods and means of the direction "Relativistic geodesy";

- planning the use of the achievements of ground-based and space laser gravitationalwave astronomy in gravimetry. The creation of laser gravitational-wave antennas can be considered a locomotive in the development of laser gradient-metric systems. The margin for the achievable accuracy of laser interferometers based on the achievements of laser gravitational-wave antennas is 5-6 orders of magnitude, which is a significant incentive for the development of optical means of ground and space geodesy.

\section{References}


1. B. Tapley, S. Bettadpur, M. Watkins, C. Reigber, The Gravity Recovery and Climate Experiment: Mission overview and early results, Geophysical Research Letters 31(9):4 (2004) DOI:10.1029/2004GL019920

2. M. Drinkwater, R. Floberghagen, R. Haagmans, D. Muzi, A. Popescu, GOCE: ESA's first earth explorer core mission, Space Science Reviews, 108, pp. 419-432, (2003) DOI:10.1023/A:1026104216284

3. D. Beggan, S. Macmillan, J. Brown, J. Grindrod, Quantifying Global and Random Uncertainties in High Resolution Global Geomagnetic Field Models Used for Directional Drilling, SPE Drilling \& Completion, pp. 1-10, (2021) DOI:10.2118/204038-pa

4. B. Meyer, A. Chulliat, R. Saltus, Derivation and Error Analysis of the Earth Magnetic Anomaly Grid at 2 Arc-Minute Resolution Version 3 (EMAG2v3), Geochemistry, Geophysics, Geosystems, 18, (2017) DOI:10.1002/2017GC007280.

5. http://icgem.gfz-potsdam.de/tom_longtime

6. Earth parameters 1990. Reference document. Moscow: Military Topographic Directorate of the General Staff of the Armed Forces of the Russian Federation, 2018.

7. https://cgkipd.ru/opendata/gao2012/

8. O.V. Denisenko, I.S. Silvestrov, V.F. Fateev, R.A. Davlatov, Possibilities of forming a unified geocentric coordinate system, Almanac of modern metrology, No. 15, pp. 92112, (2018) (In Russian)

9. L.S. Sugaipova, Development and research of methods for multi-scale modeling of the geopotential, Dissertation for the degree of Doctor of Technical Sciences: 25.00.32, MIIGAiK, Moscow, 325 p., (2018) (In Russian)

10. D.S. Bobrov, Development of methods and means for creating navigational gravity maps, Dissertation for the degree of Candidate of Technical Sciences, All-Russian Scientific Research Institute of Physical-Technical and Radio Engineering Measurements, Mendeleevo, (2020) (In Russian)

11. A.P. Yuzefovich, Gravity field and its study, M: Publishing house MIGAiK, 192 p., (2014) (In Russian)

12. https://usgs.gov/centers/eros/science/usgs-eros-archive-digital-elevation-shuttle-radartopography-mission-srtm-1-arc?qt-science_center_objects $=0 \#$ qtscience center_objects

13. https://www.unb.ca/fredericton/engineering/depts/gge/resources.html

14. D.S. Bobrov, Investigation of the informativeness of the gravity field for indoor navigation, Scientific Assembly of the International Association of Geodesy, June 28 July 2, Beijin, China (2021)

15. http://microglacoste.com/

16. I.A. Bunin, E.N. Kalish, D.A. Nosov, M.G. Smirnov, Yu.F. Stus, Field absolute laser ballistic gravimeter, Avtometriya, V. 46, No. 5, pp. 94-102, (2010) (In Russian)

17. L.F. Vitushkin, F.F. Karpeshin, E.P. Krivtsov, P.P. Krolitsky, V.V. Nalivaev, O.A. Orlov, M.M. Khaleev, State primary special standard of acceleration for gravimetry GET 190-2019, Measuring equipment, No. 7, pp. 3-8, (2020), (In Russian) DOI: https://doi.org/10.32446/0368-1025it.2020-7-3-8

18. https://scintrexltd.com/product/cg-6-autograv-gravity-meter/

19. A.A. Golovan, V.V. Klevtsov, I.V. Koneshov, Yu.L. Smoller, S. Sh. Yurist, Peculiarities of using the gravimetric complex GT-2a in problems of airborne gravimetry, Physics of the Earth, No. 4, pp. 127-134, (2018) 
20. A. Krasnov, A. Sokolov, L. Elinson, A new air-sea shelf gravimeter of the Chekan series, Gyroscopy and Navigation, 5, pp. 131-137, (2014), DOI:10.1134/S2075108714030067

21. P. Gillot, B. Cheng, A. Imanaliev, S. Merlet, F. Pereira dos Santos, The LNE-SYRTE cold atom gravimeter, 1-3, (2016), DOI:10.1109/EFTF.2016.7477832

22. Zhou Min-Kang, Duan Xiao-Chun, Chen Le-Le, Luo Qin, Xu Yao-Yao, Hu ZhongKun, Micro-Gal level gravity measurements with cold atom interferometry, Chinese Physics B, 24(5): 050401, (2015), DOI: 10.1088/1674-1056/24/5/050401.

23. X. Wu, Z. Pagel, S. Bola, T. Nguyen, F. Zi, D. Scheirer, H. Müller, Holger, Gravity surveys using a mobile atom interferometer. Science Advances, 5, eaax0800, (2019), DOI:10.1126/sciadv.aax0800.

24. M.S. Aleinikov, V.N. Baryshev, I.Yu. Blinov, D.S. Kupalov, G.V. Osipenko, Prospects for the development of a sensitive atomic interferometer based on cold rubidium atoms, Measuring equipment, No. 7, pp. 9-12, (2020) DOI: https://doi.org/10.32446/0368-1025it.2020-7- 9-12 (In Russian)

25. C. Hirt, Automatic determination of vertical deflections in real time by combining GPS and digital zenith camera for solving the GPS-height-problem, Proc., 14th Int. Technical Meeting of the Satellite Division of the Institute of Navigation, Institute of Navigation, Alexandria, 2540-2551, (2001)

26. M.M. Murzabekov, V.F. Fateev, A.V. Pruglo, S.S. Ravdin, The results of astromeasurements of the deflection of vertical using a new measurement method, Almanac of modern metrology, No. 2 (22), pp. 42-56, (2020) (In Russian)

27. Method for measuring the deflection of vertical and a device for its implementation: US Pat. 2750999 Rus. Federation. No. 2020139586; declared 12/01/20; publ. 07.07.21, Bul. No. 19

28. R. Eötvös, D. Pekár, E. Fekete, Beiträge zum Gesetze der Proportionalität von Trägheit und Gravität, Annalen der Physik, 373, 11-66, (2006), DOI:10.1002/andp.19223730903

29. L. Volgyesi, G. Szondy, G. Péter, B. Kiss, G. Barnafoldi, L. Deák, É. Csaba, E. Fenyvesi, G. Grof, L. Somlai, P. Harangozó, P. Levai, P. Ván, Remeasurement of the Eötvös experiment - status and first results, 042, (2019), DOI:10.22323/1.353.0042.

30. Resolution of the International Association of Geodesy No. 1 "On the definition and implementation of the international reference system of heights", Prague, Czech Republic, June 22 - July 2, 2015. http://iag.dgfi.tum.de/fileadmin/IAGdocs/IAG_Resolutions_2015.pdf.

31. J. Müller, D. Dirkx, S.M. Kopeikin, G. Lion, High Performance Clocks and Gravity Field Determination, Space Sci Rev 214:5, (2018), DOI 10.1007/s11214-017-0431-z.

32. L.D. Landau, E.M. Lifshits, Field theory, -M.: Nauka, 460 p., (1967) (In Russian)

33. V.F. Fateev, Relativistic metrology of near-earth space-time, Monograph, Mendeleevo: FSUE "VNIIFTRI", 439 p., (2017) (In Russian)

34. G. Jacopo, K. Silvio, V. Stefan, H. Sebastian e.a. Geodesy and metrology with a transportable optical clock, Nature Physics, 14, (2018), DOI:10.1038/s41567-0170042-3.

35. T. Masao, U. Ichiro, O. Noriaki, Y. Toshihiro, K. Kensuke, S. Hisaaki, K. Hidetoshi, Test of general relativity by a pair of transportable optical lattice clocks, Nature Photonics, 14, 1-5, (2020), DOI:10.1038/s41566-020-0619-8. 
36. V.F. Fateev, A.I. Zharikov, V.P. Sysoev, E.A. Rybakov, F.R. Smirnov, Measurement Of The Difference In The Earths Gravitational Potentials With The Help Of A Transportable Quantum Clock, Doklady Earth Sciences, 472(1): 91-94, (2017), DOI:10.1134/S1028334X17010147

37. V. F. Fateev, E. A. Rybakov, Experimental Verification of the Quantum Level on a Mobile Quantum Clocks, Doklady Physics, 66(1): 17-19, (2021), DOI:10.1134/S1028335820110038

38. V.F. Fateev, E.A. Rybakov, F.R. Smirnov, A Method Of Relativistic Synchronization Of Moving Atomic Clocks And Experimental Verification Thereof, Technical Physics Letters, 43(5): 456-459, (2017), DOI:10.1134/S1063785017050182

39. V.F. Fateev, Yu.F. Smirnov, A.I. Zharikov, E.A. Rybakov, F.R. Smirnov, $A$ Relativistic Synchronization-Based Experiment for Improving the Accuracy of Time Scale Transmission, Technical Physics Letters, 47(1): 35-37, (2021), DOI:10.1134/s1063785021010065

40. R. Kornfeld, B. Arnold, M. Gross, N. Dahya, W. Klipstein, P. Gath, S. Bettadpur, GRACE-FO: The Gravity Recovery and Climate Experiment Follow-On Mission, Journal of Spacecraft and Rockets, 56(3):931-951, (2019), DOI:10.2514/1.A34326

41. N.F. Klyuev, V.F. Fateev, L.L. Ilyin, N.A. Byrkov, I.V. Sakhno, Principles of constructing two-position space-based SARs, Materials of the military-scientific conference on March 21-23, 1995, St. Petersburg: VIKKA,.V. 2, pp. 335-338, (1996) (In Russian)

42. M.P. Clarizia, C.P. Gommenginger, S.T. Gleason, M.A. Srokosz, C. Galdi, M. Bisceglie, Analysis of GNSS-R delay-Doppler maps from the UK-DMC satellite over the ocean, Geophysical Research Letters 36(2), (2009), DOI:10.1029/2008GL036292

43. M. Antoniou, M. Cherniakov, GNSS-based bistatic SAR: a signal processing view, EURASIP J. Adv. Signal Process 2013(1), (2013), DOI:10.1186/1687-6180-2013-98

44. J. Wickert et, al., GEROS-ISS: GNSS REflectometry, Radio Occultation, and Scatterometry Onboard the International Space Station, IEEE Journal of Selected Topics in Applied Earth Observations and Remote Sensing 9(10):4552-4581, (2013), DOI:10.1109/JSTARS.2016.2614428

45. W. Li, E. Cardellach, F. Fabra, S. Ribó, A. Rius, Lake Level and Surface Topography Measured with Spaceborne GNSS-Reflectometry from CYGNSS Mission: Example for the Lake Qinghai. Geophysical Research Letters, 45(24), (2018), DOI:10.1029/2018GL080976

46. V.F. Fateev, A.V. Ksendzuk, P.S. Obukhov et al., Experimental bistatic radar complex, Electromagnetic waves and electronic systems, No. 5, V.17, pp. 58-61, (2012) (In Russian)

47. A. Schleicher, N. Brandt, S. Vitale, D. Bortoluzzi, Control Tasks and Functional Architecture of the LISA Pathfinder Drag-Free System, Proc. of the 6th Intern. ESA Conference on Guidance, Navigation and Control Systems, Loutraki, Greece, October 17-20, 2005 (ESA SP-606, January 2006)

48. L. Duchayne, F. Mercier, P. Wolf, Orbit determination for next generation space clocks, Astronomy and Astrophysics 504(2), (2007), DOI:10.1051/00046361/200809613

49. V.F. Fateev, V.P. Lopatin, Space bistatic radar for monitoring the ocean surface profile based on GNSS signals, Izvestiya vuzov. Instrument making, V. 62, No. 5. pp. 484-491, (2019) (In Russian) 
50. V.F. Fateev, R.A. Davlatov, Analysis of the capabilities of a space gradiometer on free masses, Almanac of modern metrology, No. 2 (22), pp. 65-72, (2020) (In Russian)

51. O.V. Denisenko, I.S. Silvestrov, V.F. Fateev, R.A. Davlatov, Laser space gravity gradiometer, Positive decision on the application for a patent for invention No. 2021102273 dated 01.02.2021 (In Russian)

52. M. Enrico, Time, Atomic Clock, and Relativistic Geodesy, Munchen, p. 127, (2013)

53. P. Delva, N. Puchades, E. Schönemann et al., Gravitational Redshift Test Using Eccentric Galileo Satellites, Physical Review Letters 121(23), (2018), DOI:10.1103/PhysRevLett.121.231101

54. S. Herrmann, F. Finke, M. Lulf et .al., Test of the Gravitational Redshift with Galileo Satellites in an Eccentric Orbit // Physical Review Letters 121(23), (2018), DOI:10.1103/PhysRevLett.121.231102

55. L.D. Landau, E.M. Lifshits, Continuous media electrodynamics, 2 nd ed., Rev. and add. Moscow.: Nauka, 621 p. (1982) (In Russian)

56. V.F. Fateev, Refractive properties of the Earth's gravitational sphere in rotating reference frames, Electromagnetic waves and electronic systems, 18, No. 5, pp. 073082, (2013) (In Russian)

57. V.F. Fateev, Relativistic metrology of near-earth space-time and its practical applications, Astron. Rep., 62(12):1036-1041, (2018)

https://doi.org/10.1134/S1063772918120041

58. A. Abramovici, W.E. Althouse, R.W.P. Drever et al., LIGO: The Laser Interferometer Gravitational-Wave Observatory, Science 256(5055):325-336 (1992), DOI:10.1126/science.256.5055.325

59. R. Flaminio et al., The gravitational wave detector VIRGO, http://icfananobeam.web.cern.ch/icfa-nanobeam/paper/Flaminio_Virgo.pdf.

60. B. Willke, P. Aufmuth, C. Aulbert et al., The GEO600 gravitational wave detector, Classical and Quantum Gravity 19(7):1377-1387, (2002), DOI:10.1088/02649381/19/7/321

61. Y. Aso et al., Interferometer design of the KAGRA gravitational wave detector, Physical review D: Particles and fields 88(4) (2013),

DOI:10.1103/PhysRevD.88.043007

62. V.F. Fateev, R.A. Davlatov, Space-Based Gravitational-Wave Detectors: Development of Ground-Breaking Technologies for Future Space-Based Gravitational

Gradiometers, Astronomy Reports, 63(8):699-709, (2019)

DOI:10.1134/S1063772919080018

63. H. James et al., LISA: laser interferometer space antenna for gravitational wave measurements, Classical and Quantum Gravity, V.13, A247-A250, (1996).

64. N. Seto, S. Kawamura, T. Nakamura, Possibility of direct measurement of the acceleration of the universe using laser interferometer gravitational wave antenna in space, Physical Review Letters 87(22), (2001) DOI:10.1103/PhysRevLett.87.221103

65. L. Jun et al., TianQin: a space-borne gravitational wave detector, Classical and Quantum Gravity 33(3), (2015) DOI:10.1088/0264-9381/33/3/035010

66. J.W. Conclin et al., LAGRANGE: LAser GRavitational-wave ANtenna at GEo-lunar Lagrange points, (2011)

67. M. Tinto, J.C.N. de Araujo, O.D. Aguiar, M.E.S. Alves, Searching for gravitational waves with a geostationary interferometer, Astroparticle Physics, 48, (2013) DOI:10.1016/j.astropartphys.2013.07.001 
68. V.I. Pustovoit, S.I. Donchenko, O.V. Denisenko, V.F. Fateev, The concept of creating a space laser gravitational antenna in the geocentric orbit GLONASS "SOIGA", Almanac of modern metrology, 1(21), pp. 27-49, (2020) (In Russian) 